\title{
Infective endocarditis or myxoma? Description of a patient with new diagnosis of congestive heart failure
}

\section{Endocardite infettiva o mixoma? Descrizione di un caso con scompenso cardiaco di nuova insorgenza}

\author{
Marzia Testa2, Enrico Lombardo1, Enrico Avogadri1, Marco Agostini3, \\ Giuseppe Forte ${ }^{4}$, Salvatore Piccolo1, Mauro Feola1
}

\begin{abstract}
Infective endocarditis or myxoma? Description of a patient with new diagnosis of congestive heart failure. M. Testa, E. Lombardo, E. Avogadri, M. Agostini, G. Forte, S. Piccolo, M. Feola.

ABSTRACT: Infective endocarditis (IE) is an inflammatory disease which interests heart endothelium and mostly heart valves. IE is not a uniform disease, but presents in a variety of different forms that makes the diagnosis dif-
\end{abstract}

ficult. Echocardiography is a crucial diagnostic tool for the diagnosis, especially in those patients who have no typical symptoms as in the case here presented, in which the possibility of a myxoma was also considered.

Key words: infective endocarditis, myxoma, intracardiac mass, echocardiography.

Monaldi Arch Chest Dis 2012; 78: 107-108.

\footnotetext{
${ }^{1}$ Cardiovascular Rehabilitation-Heart Failure Unit, Ospedale SS Trinita' Fossano Italy.

2 SCDU Geriatria e Malattie Metaboliche dell' Osso, Torino, Italy.

${ }_{3}^{3}$ Cardiosurgery Division, Ospedale S. Croce-Carle Cuneo Italy.

4 Pathology Service, Ospedale S. Croce-Carle Cuneo Italy.
}

Corresponding author: Mauro Feola; Cardiovascular Rehabilitation-Heart Failure Unit, Ospedale SS Trinita' Fossano, CN, Italy; E-mail address: m feola@virgilio.it; +39/0172699365

\section{Introduction}

Infective endocarditis (IE) is an inflammatory disease which interests heart endothelium and mostly heart valves. IE is not a uniform disease, but presents in a variety of different forms that makes the diagnosis difficult. Echocardiography is an important diagnostic tool for the diagnosis, especially in those patients who have no typical symptoms as in the case here presented, in which the possibility of an intracardiac mass (myxoma or fibroelastoma) was also considered.

\section{Case report}

A 69 year-old woman was admitted to our hospital with mild shortness of breath on exertion and symptoms of congestive heart failure. On physical examination her temperature was $35.6^{\circ} \mathrm{C}$, her blood pressure was measured $180 / 105 \mathrm{mmHg}$, the heart frequency 90 beats/min and no abnormalities were detected in routine laboratory tests. A mild mitral regurgitation murmur was heard for the first time at cardiac examination with a normal first tone sound. Moreover, the anamnesis for cardiovascular risk factors proved to be negative as well as the history of prolonged fever or surgical procedures at risk for developing endocarditis. To assess cardiac function and investigate on the mitral regurgitation, a transthoracic echocardiogram (TTE) was performed revealing a mass on mitral valve detected by chance, together with a moderate mitral regurgitation (vena contracta $0.4 \mathrm{~cm}$ ). The transesophageal echocardiography (TEE) showed a mobile mass of $15 \times 10 \mathrm{~mm}$ on the anterior mitral leaflet, highly suspected to be a myxoma or a fibroelastoma (Fig. 1). The absence of calcification seemed to exclude a tumor-like mitral calcification. The laboratory research of infective

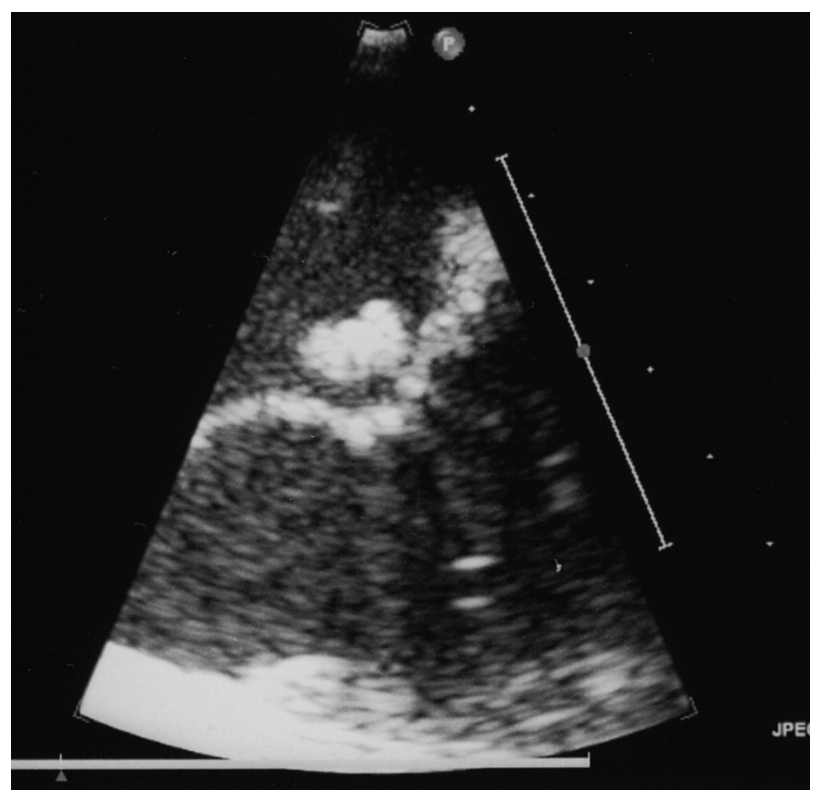

Figure 1. - TransEsophageal Echocardiography shows a mobile mass of $15 \times 10 \mathrm{~mm}$ on the anterior mitral leaflet. 


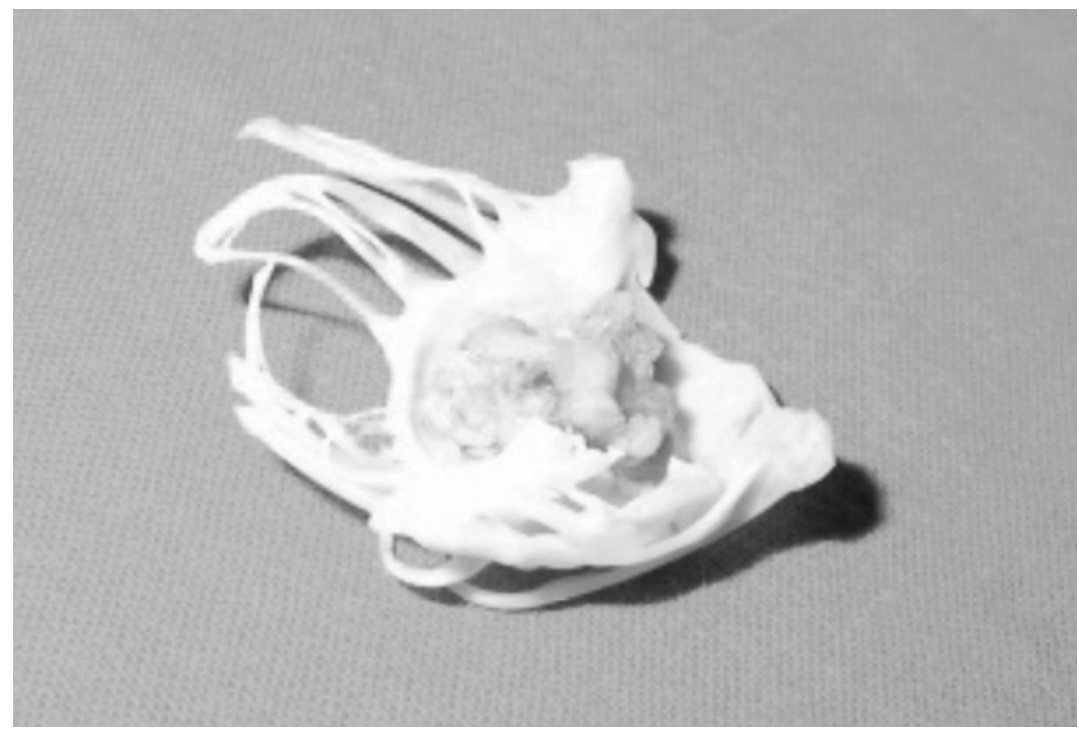

Figure 2. - Grossly, the mitral valve shows an irregular vegetation, sessile, measuring $1.5 \mathrm{~cm}$, composed of gray-whitish tissue of calcified appearance.

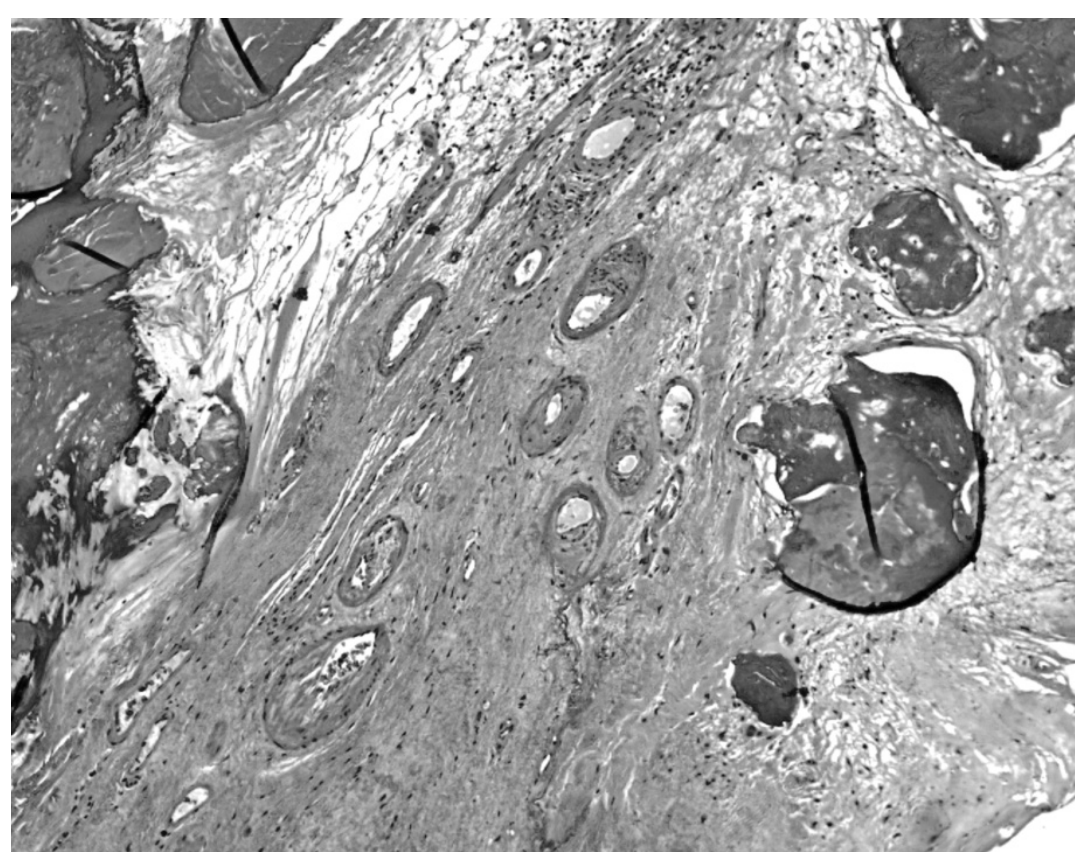

Figure 3. - On microscopic examination (Haematoxylin-Eosin) the mitral valve shows fibrous thickening of the wall with calcification and vascular abnormalities, such a collection of thickwalled dilatated vessels with a fibromuscolar wall, lined by endothelial cells and embedded in a fibrous stroma: this sign may be found as result of an endocarditis. infective endocarditis frequently develops in absence of underlying structural heart disease [2] and its clinical course is more insidious: in fact, the development of fever or other classic clinical hallmarks of IE seemed to be less manifested.

Transesophageal echocardiography (TEE) represents an important step for the evaluation of vegetations [3], but it should be considered that IE could mimic intracardiac tumors, especially myxomas or fibroelastoma. The fibroelastoma is a typically small $(1-2 \mathrm{~cm})$, non vascularized, benign tumor that represents $8 \%$ of primitive cardiac tumors (4). The main site of development has been described on valvular endocardium (aortic valve or atrioventricular valves) and only rarely has been observed on papillary muscles. The cardiac mixoma, howewhere, is the most frequent benign primitive cardiac tumor. Cardiac mixomas represented the $78 \%$ of surgical treated tumor of the heart (5), being observed in adults (mean age 50 years old) with the preference of female gender (1.8/1). The cardiac mixoma has been classically considered a benign neoplasm but two complications might influence severely the clinical prognosis: the mixoma embolization and the transvalvular obstruction due to dislocation of the mass into the orifice.

In our case report, we considered all possibilities and only the surgical biopsy specimen could resolve the clinical suspicion. Moreover, the decision for surgery was established on TEE findings, that underlined the high risk of embolization and on the clinical presentation (congestive heart failure) that suggested a replacement reconstruction of the mitral valve [3]. endocarditis using three venous blood cultures was negative as well as the inflammatory markers (C Reactive protein $<0.5 \mathrm{mg} / \mathrm{dl}$, white cells count $<6000 / \mathrm{ml}$ ). The patient underwent mitral valve replacement with a biological prosthetic valve, according to the high risk for systemic embolization. The pathologic examination excluded the presence of atrial myxoma or fibroelastoma, identified a complex matrice that might be the result of a subclinical, silent endocarditis (Fig. 2, 3).

\section{Discussion}

The variability of clinical presentation of infective endocarditis (IE) continues to make the diagnosis difficult [1]. In addition, in older patients

\section{References}

1. Murdoch DR, Corey GR, Hoen B, et al. International Collaboration on Endocarditis-Prospective Cohort Study (ICEPCS) Investigators. Clinical presentation, etiology, and outcome of infective endocarditis in the 21st century: the International Collaboration on Endocarditis-Prospective Cohort Study. Arch Intern Med. 2009 Mar 9; 169(5): 463-73.

2. Gregoratos G. Infective endocarditis in thee elderly: diagnosis and management. Am J Geriatr Cardiol. 2003 MayJun; 12(3): 183-9.

3. Habib G. Infective endocarditis: what's new? European Society of Cardiology (ESC) Guidelines 2009 on the prevention, diagnosis and treatment of infective endocarditis. Presse Med. 2010 Jun; 39(6): 704-9.

4. Basso C, Bottio T, Valente M, Bonato R, Casarotto D, Thiene G. Primary cardiac valve tumors. Heart 2003; 89: 1259-60.

5. Blondeau P. Primary cardiac tumors-French studies of 533 cases. Thorac Cardiovasc Surg 1990; 38: 192-5. 